\title{
Long-term outcomes of tension-free repair for the primary inguinal hernias
}

\author{
Fatih Büyüker®, Hakan Baysalø, Salih Tosun $\odot$ \\ Department of General Surgery, Medeniyet University School of Medicine, Istanbul, Turkey
}

\section{ABSTRACT}

Objectives: Mesh repair is the gold standard in inguinal hernia(IH) repair. If suture repair is to be performed, the common view is to apply tension-free repair. The aim of our study was to analyze retrospectively the changes in primary IH repair at our clinic in 24-years period with regard to the surgical techniques used, patient demographics, complications, and duration of hospital stay.

Methods: The study is based on retrospective analyses of IH repair in 1020 patients in two different periods. In the first period of the study, between 1997-1999 Modified Bassini (MB) method was used for suture repair and Lichtenstein (LH) method was used for mesh repair. In the second period of the study, between 20172019, LH method was used for mesh repair and Posterior Wall Darn (PWD) method was used for suture repair. Results: The rates of postoperative complications were high and time to return to work was longer in suture repair with MB. Less postoperative complications, shorter time to return to work and less recurrence were observed in the PWD method compared to MB.

Conclusions: Suture repair is a preferable option only in relevant cases and these must be tension free repair techniques such as PWD. When required, suture repairs could be successfully performed with low complication and recurrence rates, similar to mesh repairs by experienced surgical teams.

Keywords: tension-free, posterior wall darn, hernia repair

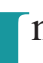
nguinal hernia (IH) repair is one of the most common performed operations worldwide. An average of 20 million people undergo IH surgery each year [13]. It is the most common abdominal wall hernia, accounting for approximately $75 \%$ of all hernias, and there is a $27 \%$ lifelong recurrence risk of IH in men and $3 \%$ in women [4]. Though performed so frequently, there is no consensus on which surgical method is the best in IH repair yet. Being the pioneer of the modern hernia surgery, Bassini suture repair is still applied in the world with many modifications [5, 6]. Mesh repair techniques in IH repair that started with the Liectenstein method have gradually decreased the preference of suture repairs, along with the facts that the availability of the mesh become easier and studies have shown that the recurrence rate in mesh repair is lower [7].

The aim of current study was to analyze the changes in IH repair at our clinic in 20-years period with regard to the surgical techniques used, patient demographics, complications, and duration of hospital stay retrospectively.

\section{METHODS}

The study is based on retrospective analyses of IH repair in 1020 patients at a single center in two differ- 
ent periods.

In the first period of the study, the results of unilateral primary inguinal hernia surgeries performed at our clinic between the years 1997-1999 were evaluated. The patients were divided into 2 groups as being performed suture and mesh repair. Modified Bassini (MB) method was used for suture repair and Lichtenstein (LH) method was used for mesh repair.

In the second period of the study, operations performed for unilateral primary inguinal hernia between the years 2017-2019 were analyzed. LH method was used for mesh repair and Posterior Wall Darn (PWD) method was used for suture repair.

In the first period, surgical methods were determined according to the surgeon's and patient's preferences and the mesh availability. In the second period, suture repair was used for patients who refused the mesh or patients younger than 45 years or patients with contaminated wounds. Standard LH repair was applied to the remaining patient group.

Exclusion criterias were as follows:

- Laparoscopic repairs (TAPP)

- Endoscopic repairs (TEP)

- Femoral hernias

- Obturator hernias

- Recurrent cases and

- Bilateral hernias

The demographic characteristics, post-operative length of stay, postoperative complications, and recurrence rates of the patients were analyzed.

\section{Surgical Methods}

\section{Modified Bassini Technique (MB)}

With an oblique inguinal incision, the skin, subcutaneous, superficial fascia, and external oblique aponeurosis were dissected. The medial leaf of the external oblique aponeurosis was dissected up to the rectus muscle. The lateral leaf was dissected until the Poubart ligament and the iliopubic tract were exposed. In indirect hernias, the hernia sac was dissected to the neck level, ligated and excised (high-ligation). In direct hernias, the protruded pouch was inverted into the abdomen with purse-string sutures. Reinforcement sutures were placed seperately, at intervals of one centimeter, starting from the pubis with prolene suture material, passing through the conjoint tendon and the poubart ligament. The inner ring has been narrowed up to allow the passage of finger pulp (Fig. 1).

\section{Lichtenstein Technique (LH)}

The dissection was performed until the Poubart ligament and iliopubic tract were exposed. The pubic bone was exposed up to approximately $2 \mathrm{~cm}$ medial of the pubic tubercle. A monofilament polypropylene mesh with $8 \times 15 \mathrm{~cm}$ size was used for the repair. The medial side of the mesh was suture to the aponeurotic tissue above the pubic bone, covering the pubic bone 2-3 cm medial with 3-0 prolene suture. With the same suture, the lower edge of the mesh was suture to the poubart ligament continuously and not too tightly, and the procedure was continued up to lateral of the internal ring. An incision was made on the lateral edge of the mesh and 2 tails were created with the upper part wider than the lower side. The upper edge of the mesh was continuously suture with nonabsorbable suture to

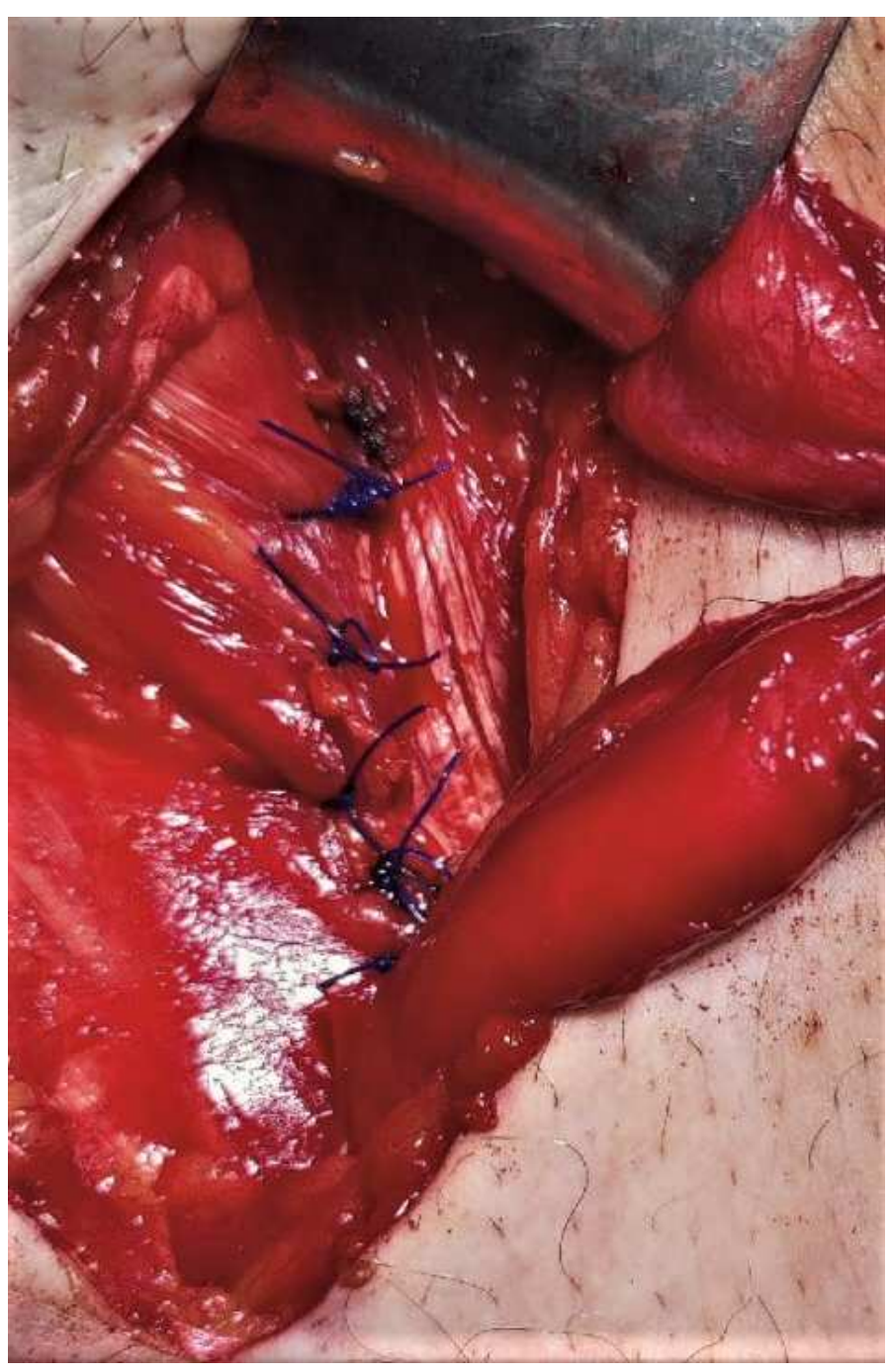

Fig. 1. MB repair with seperated sutures. 
internal oblique aponeurosis, $2-3 \mathrm{~cm}$ beyond the borders of Hasselbach's triangle. The lower edges of both tails were attached to the inguinal ligament and just lateral to the underlying continuous suture with a few nonabsorbable sutures. An excess of 3-4 $\mathrm{cm}$ mesh that was left on the lateral edge of the mesh, was folded under the eksternal oblique aponeurosis which would cover the mesh (Fig. 2).

\section{Posterior Wall Darn Method (PWD)}

In this method, sutures were placed between the conjoint tendon and the iliopubic tract over 2 layers of polypropylene so that the stitches weren't be too tight, and rectus muscle and fascia were not stretched. Sutures were passed through both the iliopubic tract and the conjoint tendon at equal intervals of at least $6 \mathrm{~mm}$ and at most $12 \mathrm{~mm}$ length. Unlike the Bassini technique, the tissues were not tightly brought close to each other, only the repair line was attached tightly and the suture was left loose in accordance with the PWD technique. After narrowing the internal orifice of the inguinal canal, the suture is locked and tied (Fig. $3)$.

\section{Statistical Analysis}

Statistical analysis was performed using the SPSS for Windows version 22.0 software (SPSS Inc., Chicago, IL, USA). The Kolmogorov-Smirnov test

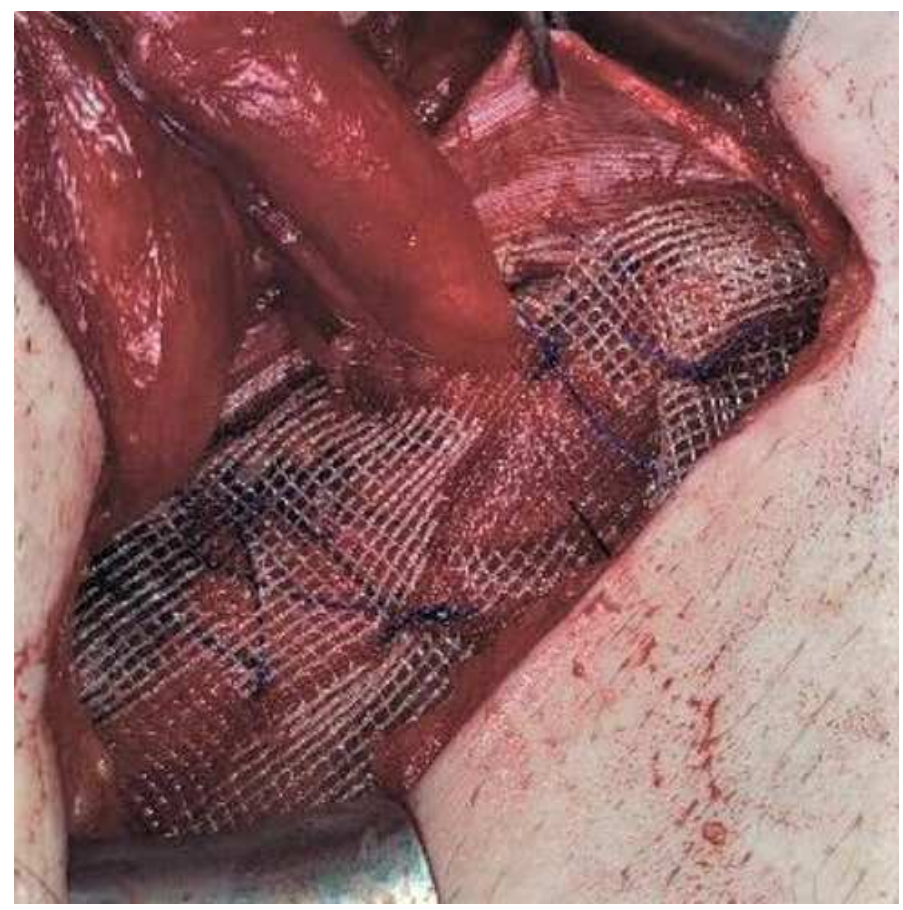

Fig. 2. LH repair with mesh.

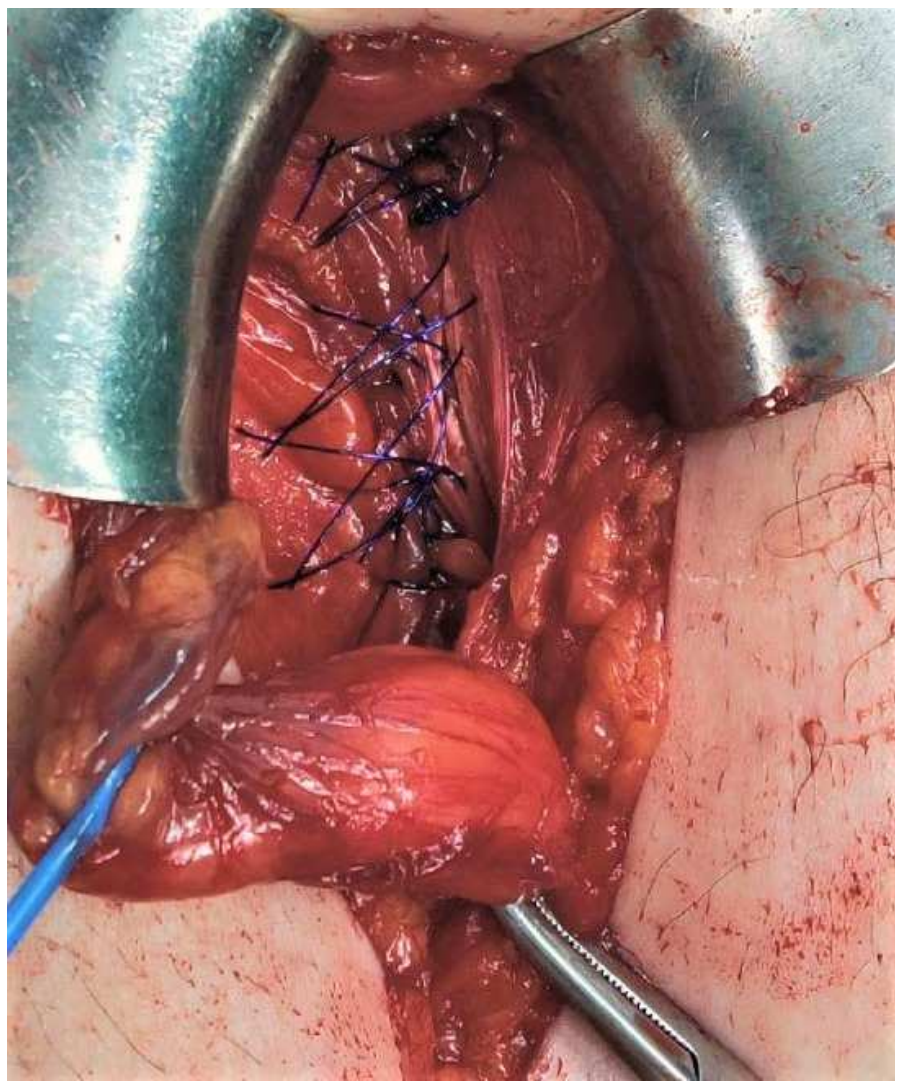

Fig. 3. PWD tension-free repair with continuous sutures.

was used to test the normal distribution of data. Descriptive data were expressed in mean \pm standard deviation, medians (range) and number and frequency $(\%)$.

\section{RESULTS}

At the first period, there were 150 primary unilateral inguinal hernia cases within 2-years time. LH technique was applied to $90(60 \%)$ patients and $\mathrm{MB}$ technique was applied to $60(40 \%)$ patients. $79(88 \%)$ of the patients in the LH group and $52(87 \%)$ of the patients in the MB group were male. Most of the patients $(75 \%)$ were $\leq 45$ years old. $67(75 \%)$ of the patients who underwent LH repair and $45(75 \%)$ of the patients in the MB group were $\leq 45$ years old. After the LH repair performed at this period, the postoperative hospital stay was approximately 1 day (6-28 hours), and the average time to return to work was 10 days (7-16 days). Postoperative surgical site infection was developed in only $2(2.2 \%)$ patients in the LH repair group and early recurrence was not observed. (Table 1). After the MB repair performed at this pe- 
riod, the average postoperative hospital stay was 3 days (25-96 hours), and the average time to return to work was 22.5 days (17-30 days). In the MB group, 3 $(5 \%)$ patients had developed surgical site infection; 2 $(3.3 \%)$ had scrotal hematoma; and $2(3.3 \%)$ had early recurrence.

At the second period, there were 870 primary unilateral inguinal hernia cases within 2 years. LH technique was applied to 792 (91\%) and PWD technique was applied to $78(19 \%)$ patients respectively. 811 (93\%) patients were male and $59(7 \%)$ were female. $665(84 \%)$ of the patients who underwent LH repair are were $>45$ years old, while $66(85 \%)$ of the patients who underwent PWD repair were $\leq 45$ years old. The mean postoperative hospital stay following the LH repair performed at this period was 1.01 days (11-172 hours), and the average time to return to work was 8.9 days (4-18 days). In the LH repair group, 17 (2.1\%) patients developed post-operative seroma, $21(2.7 \%)$ patients developed surgical site infection, and 13 $(1.6 \%)$ patients developed scrotal hematoma. Early recurrence was observed in $11(0.9 \%)$ patients in LH repair group. The mean postoperative hospital stay in PWD repair group was 1 day (7-49 hours), and the time to return to work was 9.2 days (3-19 days) on average. In the PWD repair group, post-operative complications were; seroma in $3(3.8 \%)$ patients, surgical site infection in $4(5.1 \%)$ patients, and scrotal hematoma in $4(5.1 \%)$ patients. Early recurrence was observed in $1(1.3 \%)$ patient in PWD repair group (Table 1).

\section{DISCUSSION}

With the Bassini method, an important milestone

Table 1. Demographic data and clinical outcomes of patients

\begin{tabular}{|c|c|c|c|c|}
\hline & \multicolumn{2}{|c|}{$\begin{array}{c}1^{\text {st }} \text { PERIOD } \\
(n=150)\end{array}$} & \multicolumn{2}{|c|}{$\begin{array}{c}2^{\text {nd }} \text { PERIOD } \\
(n=870)\end{array}$} \\
\hline & Lichtenstein & $\begin{array}{c}\text { Modified } \\
\text { Bassini } \\
\end{array}$ & Lichtenstein & $\begin{array}{c}\text { Posterior } \\
\text { Wall Darn } \\
\end{array}$ \\
\hline Number of patients (\%) & $90(60 \%)$ & $60(40 \%)$ & $792(91.0 \%)$ & $78(9.0 \%)$ \\
\hline \multicolumn{5}{|l|}{ Gender, n (\%) } \\
\hline Male & $79(87.8 \%)$ & $52(86.7 \%)$ & $739(93.3 \%)$ & $72(92.3 \%)$ \\
\hline Female & $11(12.2 \%)$ & $8(13.3 \%)$ & $53(6.7 \%)$ & $6(7.7 \%)$ \\
\hline \multicolumn{5}{|l|}{ Age, n (\%) } \\
\hline $16-25$ years & $17(18.8 \%)$ & $9(15.0 \%)$ & $9(1.1 \%)$ & $22(28.2 \%)$ \\
\hline $26-35$ years & $28(31.1 \%)$ & $17(28.3 \%)$ & $27(3.4 \%)$ & $35(44.9 \%)$ \\
\hline $36-45$ years & $22(24.4 \%)$ & $19(31.7 \%)$ & $105(13.3 \%)$ & $9(11.5 \%)$ \\
\hline $46-65$ years & $14(15.5 \%)$ & $9(15.0 \%)$ & $411(51.9 \%)$ & $10(12.8 \%)$ \\
\hline $66-75$ years & $6(6.6 \%)$ & $4(6.7 \%)$ & $179(22.6 \%)$ & $1(1.3 \%)$ \\
\hline$>75$ years & $3(3.3 \%)$ & $2(3.3 \%)$ & $61(7.7 \%)$ & $1(1.3 \%)$ \\
\hline $\begin{array}{l}\text { Postoperative hospital stay }(\mathrm{h}) \text {, } \\
\text { median (range) }\end{array}$ & $24.0(6-28)$ & $72.0(25-96)$ & $24.2(11-172)$ & $24.1(7-49)$ \\
\hline $\begin{array}{l}\text { Time to turn to work (d), } \\
\text { median (range) }\end{array}$ & $10.0(7-16)$ & $22.5(17-30)$ & $8.9(4-18)$ & $9.2(3-19)$ \\
\hline \multicolumn{5}{|l|}{ Postoperative complications, $\mathrm{n}(\%)$} \\
\hline Seroma & $0(0 \%)$ & $0(0 \%)$ & $17(2.1 \%)$ & $3(3.8 \%)$ \\
\hline Surgical site infection & $2(2.2 \%)$ & $3(5 \%)$ & $21(2.7 \%)$ & $4(5.1 \%)$ \\
\hline Scrotal hematoma & $0(0 \%)$ & $2(3.3 \%)$ & $13(1.6 \%)$ & $4(5.1 \%)$ \\
\hline Recurrence & $0(0 \%)$ & $2(3.3 \%)$ & $11(0.9 \%)$ & $1(1.3 \%)$ \\
\hline
\end{tabular}


in the development of modern hernia surgery, suture repair has been preferred with modifications for years and continues to be a preferred technique in selected cases [8-10]. Currently available guidelines (EHS, the HerniaSurge Group, AHS etc.) hold the view that mesh repair is the gold standard in inguinal hernia repair [11-13]. If suture repair is to be performed, the common view is to apply tension-free repair [14]. Although, in the last guideline [12], only Shouldice repair is recommended as a suture repair, different suture repair techniques are still used in the world [15].

Mesh repair was performed mainly in our clinic between the years 1997-1999, the MB technique was also applied to a significant number of patients in these years. Although early recurrence rates after MB were low, the rates of post-operative complications were high and time to return to work was longer.

In the last 20 years, the suture repair method has evolved from Bassini to its modifications like PWD, which is based on "continued non-absorbable" sutures and repair without tension [16-19]. That applied to our clinic as well. Complications such as post-operative seroma, hematoma and surgical site infection are less common that makes this method advantageous especially in contaminated cases [20]. In our cases, less post-operative complications, shorter time to return to work and less recurrence were observed in the PWD group compared to MB group.

According to HerniaSurge Group guideline; suture repair can be applied in cases such as mesh allergy and the patient's refusal to repair with mesh[12]. There are publications in the literature showing that suture repair is superior to mesh repair in terms of recurrence, in young male population with indirect hernia [21]. There are also studies indicating that young men develop more chronic pain following mesh usage compared to elderly $[22,23]$. During the past 20 years in our clinic, we have decreased using suture repair eventually, except for selected cases. In the second period; $85 \%$ of the patients who underwent PWD repair were $\leq 45$ years old and $84 \%$ of the group in which LH was applied in the second period were $>45$ years old. In the first period, the mean age was similar in the MB and LH groups. The selected case approach that we applied in our surgical routine is also have proved its effectiveness in achieving fewer complications, shorter hospital stays and shorter time to return to work in the suture repair group.
We have been using the PWD method, as a standard suture repair method in our clinic since 2000. In a study conducted by Ivanov et al. PWD was applied to 116 patients. The total complication rate was reported as $22.4 \%$ and the mean duration of hospital stay was 2.17 days [24]. In the PWD repairs that we performed in the second period in our study, our total complication rate was $10.2 \%$ and the hospital stay was around 1 day (7-49 hours). As a result of applying this method in selected cases, especially with the same experienced surgical team; the duration of hospitalization and time to return to work is shortened and post-operative complications are decreased in suture repairs.

Currently, LH repair is the standard treatment in open IH repair all over the world[11]. We perform LH repair, which we first started to apply in our clinic in 1997, more frequently today. There has been no change in the LH technique past 24 years. During this period, an important training standard has been achieved in the application of the method and the method has been a standardised procedure in our clinic. As a result of this, in the second period, a significant decrease was observed in the time to return to work.

The comparison of the two groups analysed at intervals of 24 years shows that the suture repair rates have been gradually reduced by applying to only selected cases in the second period. Thus today, recurrence rates and lenght of hospital stay are reduced and time to return to work is earlier. Furhermore, with performing suture repairs in selected cases, there are similar complication rates after mesh and suture hernia repair.

\section{CONCLUSION}

Mesh repair must be prefered in inguinal hernia treatment. Suture repair is a preferable option only in relevant cases and these must be tension free repair techniques such as PWD. When required, suture repairs could be successfully performed with low complication and recurrence rates, similar to mesh repairs by experienced surgical teams.

\section{Authors' Contribution}

Study Conception: FB, HB, ST; Study Design FB, 
HB, ST; Supervision: FB, HB, ST; Funding: FB, HB, ST; Materials: FB, HB, ST; Data Collection and/or Processing: FB, HB, ST; Statistical Analysis and/or Data Interpretation: FB, HB, ST; Literature Review: FB, HB, ST; Manuscript Preparation: FB, HB, ST and Critical Review: FB, HB, ST.

\section{Conflict of interest}

The authors disclosed no conflict of interest during the preparation or publication of this manuscript.

\section{Financing}

The authors disclosed that they did not receive any grant during conduction or writing of this study.

\section{REFERENCES}

1. Rutkow IM. Demographic and socioeconomic aspects of hernia repair in the United States in 2003. Surg Clin North Am 2003;83:1045-51.

2. Burcharth J, Pedersen M, Bisgaard T, Pedersen C, Rosenberg J. Nationwide prevalence of groin hernia repair. PLoS One 2013;8:e54367.

3. Ohene-Yeboah M, Beard JH, Frimpong-Twumasi B, Koranteng A, Mensah S. Prevalence of inguinal hernia in adult men in the Ashanti region of Ghana. World J Surg 2016;40:806-12.

4. Kingsnorth A, LeBlanc K. Hernias: inguinal and incisional. Lancet 2003;362:1561-71.

5. Ansari FM, Hameed T, Jain SK, Dua A, Nizam A. Comparison of outcome of Desarda versus Bassini repair in management of complicated inguinal hernia. Int Surg J 2019;6:3889-96.

6. Tajti J Jr, Pieler J, Ábrahám S, Simonka Z, Paszt A, Lázár G. Incarcerated gallbladder in inguinal hernia: a case report and literature review. BMC Gastroenterol 2020:14;20:425.

7. Malik AM, Khan A, Jawaid A, Laghari AA, Talpur KA. A comparative analysis between non-mesh (Bassini's) and mesh (Lichtenstein) repair of primary inguinal hernia. J Ayub Med Coll Abbottabad 2009;21:17-20.

8. Economopoulos KJ, Milewski MD, Hanks JB, Hart JM, Diduch DR. Sports hernia treatment: modified bassini versus minimal repair. Sports Health 2013;5:463-9.

9. Suradom C, Palaphun J. The usage of two umbrella mademesh plugs in herniorrhaphy: comparative study with Bassini and
Lichtenstein method. J Med Assoc Thai 2011;94:1373-9.

10. Patil SM, Gurujala A, Kumar A, Kumar KS, Mithun G. Lichtenstein mesh repair (LMR) v/s modified Bassini's repair (MBR) + Lichtenstein mesh repair of direct inguinal hernias in rural population - A comparative study. J Clin Diagn Res 2016;10:PC125.

11. Simons MP, Aufenacker T, Bay-Nielsen M, Bouillot JL, Campanelli G, Conze J, et al. European Hernia Society guidelines on the treatment of inguinal hernia in adult patients. Hernia 2009; 13:343-403.

12. HerniaSurge Group. International guidelines for groin hernia management. Hernia 2018;22:1-165.

13. Watson B, Roberts J, Dobbs B, Roberts R. Is inguinal hernia mesh safe? A prospective study. ANZ J Surg 2020;90:538-41.

14. Ran K, Wang X, Zhao Y. Open tensionless repair techniques for inguinal hernia: a meta-analysis of randomized controlled trials. Hernia 2020;24:733-45.

15. Finch DA, Misra VA, Hajibandeh S. Open darn repair vs open mesh repair of inguinal hernia: a systematic review and metaanalysis of randomised and non-randomised studies. Hernia 2019;23:523-39.

16. Memon GA, Shah SKA, Habib-Ur-Rehman. An experience with mesh versus darn repair in inguinal hernias. Pak J Med Sci 2017;33:699-702.

17. Pawlak M, Newman M, de Beaux AC, Tulloh B. The darn technique for small $(<2 \mathrm{~cm}$ diameter $)$ midline hernias. Hernia. 2020 Sep 2. doi: 10.1007/s10029-020-02283-7.

18. Olaogun JG, Afolayan JM, Areo PO, Ige JT. Repair of groin hernia under local anaesthesia in secondary health facility. ANZ J Surg 2018;88:E294-7.

19. Abd El Maksoud W, Abd El Salam M, Ahmed HH. Comparative study between Lichtenstein procedure and modified darn repair in treating primary inguinal hernia: a prospective randomized controlled trial. Hernia 2014;18:231-6.

20. Bhatti AZ, Rasool MI. Darning versus Bassini repair in primary unilateral inguinal hernia. J Coll Physicians Surg Pak 2002; $12: 169-71$.

21. Haastrup E, Andresen K, Rosenberg J. Low reoperation rates in young males after suture repair of indirect inguinal hernia: arguments for a tailored approach. Am J Surg 2017;214:844-8.

22. Aasvang E, Kehlet H. Chronic postoperative pain: the case of inguinal herniorrhaphy. Br J Anaesth 2005;95:69-76.

23. Poobalan AS, Bruce J, King PM, Chambers WA, Krukowski $\mathrm{ZH}$, Smith WC. Chronic pain and quality of life following open inguinal hernia repair. Br J Surg 2001;88:1122-6.

24. Ivanov D, Babović S, Ivanov M. Nylon-Darn herniorrhaphy: the method of choice in treatment of primary inguinal hernia. Med Pregl 2008;61:566-70. 